\title{
Parameterization and optimization of the menthol force field for molecular dynamics simulations
}

\author{
Mateusz Jasik $^{1}$ • Borys Szefczyk ${ }^{1}$ (D) \\ Received: 14 October 2015 / Accepted: 5 August 2016/Published online: 7 September 2016 \\ (C) The Author(s) 2016. This article is published with open access at Springerlink.com
}

\begin{abstract}
Menthol's various biological properties render it a useful component for medical and cosmetological applications, while its three centers of asymmetry mean that it can be used in a range of organic reactions. Menthol-substituted ionic liquids (ILs) have been found to exhibit promising antimicrobial and antielectrostatic properties, as well as being useful in organic catalysis and biochemical studies. However, so far, a force field designed and validated specifically for the menthol molecule has not been constructed. In the present work, the validation and optimization of force field parameters with regard to the ability to reproduce the macroscopic properties of menthol is presented. The set of optimized potentials for liquid simulations all atom (OPLS-AA) compatible parameters was tested and carefully tuned. The refinement of parameters included fitting of partial atomic charges, optimization of Lennard-Jones parameters, and recalculation of the dihedral angle parameters needed to reproduce quantum energy profiles. To validate the force field, a variety of physicochemical properties were calculated for liquid menthol. Both thermodynamic and kinetic properties were taken into account, including density, surface tension, enthalpy of vaporization, and shear viscosity. The obtained force field was proven to accurately reproduce the properties of the investigated compound while being fully compatible with the OPLS-AA force field.
\end{abstract}

Electronic supplementary material The online version of this article (doi:10.1007/s00894-016-3082-1) contains supplementary material, which is available to authorized users.

Borys Szefczyk

borys.szefczyk@pwr.edu.pl

1 Advanced Materials Engineering and Modelling Group, Faculty of Chemistry, Wrocław University of Technology, Wybrzeże Wyspiańskiego 27, 50-370 Wrocław, Poland
Keywords Menthol $\cdot$ OPLS-AA · Force field parameterization $\cdot$ GROMACS

\section{Introduction}

Menthol [1-methyl-4-(1-methylethyl) cyclohexan-3-ol] is a naturally occurring product, commonly obtained from the oil of plants of the Mentha genus. Its various biological and biochemical properties make it useful for medical and cosmetological applications such as antiseptics, food preservatives and as a flavouring, as well as a therapeutic agent in pain and irritation treatments [1,2]. In chemistry, its three centers of asymmetry make menthol a desirable precursor in organic synthesis. Considering also its non-toxicity, reasonable price and environmental-friendliness, menthol is used as a starting molecule in a vast number of reactions, the products of which are used in many applications. The use of menthol in organic chemistry, as well as possible applications of its derivatives, has been thoroughly reviewed elsewhere [3]. Recently, menthol has been used as a substituent in the production of several imidazolium-based ionic liquids (ILs) that present promising antimicrobial and antielectrostatic properties [4-6].

Both menthol and menthol-substituted ILs have been the subject of several studies employing computational chemistry methods - either molecular dynamics (MD) or quantum chemistry [7-13]. However, to the authors' best knowledge, none of the earlier works has developed a force field designed and validated specifically for the menthol molecule. All previous MD simulations of menthol or menthol derivatives (according to data provided by authors) were conducted with default parameters (for the respective force field), usually prepared in general for hydrocarbons or alcohols. However, especially if ILs are to be simulated, the necessity for a carefully 
prepared force field for a given molecule must be underlined [14]. The goal of this work was therefore to examine the aptitude of existing parameters in performing MD simulations of pure menthol and furthermore to check the possibility of optimizing the potential or finding a different set of parameters with better performance. The desired force field should fulfill the following requirements: (1) it should reproduce the physicochemical properties of the investigated substances, such as density, surface tension, enthalpy of vaporization or shear viscosity, with good agreement with experimental values, and (2) the force field should be compatible with the optimized potentials for liquid simulations all atom (OPLS-AA) force field $[15,16]$ that is commonly used to simulate molecular liquids.

\section{Methods}

\section{Parameterization strategy}

The aim of this work was to obtain the best possible force field for menthol compounds that would be able to reproduce their properties in a reliable way, maintaining compatibility with the OPLS-AA standard. The general strategy was to create sets of force field parameters for the menthol molecule, to test them by calculating selected physicochemical properties of liquid menthol, and, based on these calculations, to optimize the parameters to improve the accuracy of the results. The whole process was based on repeating those three operations - obtaining new parameters, calculating (recalculating) chosen properties, and tuning a part or the whole set of parameters. The variables that were changed across sets of used parameters were $\varepsilon$ and $\sigma$ from the Lennard-Jones (LJ) potential, partial atomic charges and Fourier coefficients for dihedral angles describing rotation of menthol's isopropyl and hydroxyl group, while other bonding terms were taken from the original OPLS-AA force field $[15,16]$.

\section{Reoptimization of chosen dihedral angle parameters}

The Fourier coefficients for dihedral angles were optimized for both the isopropyl and hydroxyl groups to reproduce the energy profiles calculated using quantum mechanical methods. During this procedure, the respective angle was stepped by $10^{\circ}$ in the $0-360^{\circ}$ range. The RHF/6-31G(d) energy was calculated for each conformer (while keeping the rest of the molecule fixed). The Fourier coefficients were optimized to minimize the difference between the energy calculated at Hartree-Fock level and the energy defined by force field equations:

$\sum_{a}\left(\Delta E_{f f}(a)-\Delta E_{Q M}(a)\right)=\min$

where $\alpha=0,10,20 \ldots, 360 ; E_{\mathrm{QM}}$ is the energy calculated at RHF level and the $E_{\mathrm{ff}}$ is the sum of intramolecular terms - Coulomb and van der Waals intramolecular interaction and the optimized dihedral term.

\section{Molecular systems used}

The initial molecular system for MD simulations was constructed using 219 menthol molecules, inserted into a box at random positions. The box dimensions were $3.947 \times 3.947 \times$ $3.947 \mathrm{~nm}$ (after equilibration of the system). Periodic boundary conditions were applied in all simulations. When calculating the surface tension of the system, the box was extended along the $z$ axis, so that its final length in the $z$ dimension was three times bigger than in $x / y(12 \mathrm{~nm})$. When calculating the enthalpy of vaporization, a single menthol molecule was inserted into the original, $64 \mathrm{~nm}^{3}$ box. When calculating the shear viscosity of liquid menthol, the created system contained 876 menthol molecules inserted into a box extended along the $z$ axis (final box dimension, after equilibration of the system: $3.996 \mathrm{~nm} \times 3.996 \mathrm{~nm} \times 16.382 \mathrm{~nm}$ ). For all simulations, the original OPLS-AA force field was used $[15,16]$, along with the parameters being optimized. Geometry of the system was initially optimized using the steepest-descent method, until all forces were below $200 \mathrm{~kJ} \mathrm{~mol}^{-1} \mathrm{~nm}^{-2}$. The short-range Coulomb interactions, as well as shortrange van der Waals interactions, were calculated within $1.0 \mathrm{~nm}$ cut-offs. The simulations in NPT ensemble were preceded by a preliminary equilibration run using Berendsen's thermostat and barostat [17] that lasted for $1 \mathrm{~ns}$. For the rest of the simulations (both in NVT and NPT ensembles), the NoséHoover thermostat $[18,19]$ was used. For the production run in the NPT ensemble, the Parrinello-Rahman barostat was applied [20]. All simulations were performed with a time step of $1 \mathrm{fs}$, with all bonds constrained using the LINCS algorithm [21]. A simulation time of $10 \mathrm{~ns}$ or $100 \mathrm{~ns}$ was used, depending on the calculated property (as described in "Calculations of physicochemical properties"). Uncertainties of calculated properties were estimated by dividing the production run into 200 ps blocks, and calculating averages and their standard deviations (SD) for these blocks. For the calculations, which were based on ten times longer MD simulations, the uncertainties were estimated using 2000 ps blocks. These standard deviations are presented in this work as a measure of uncertainty of the calculated properties. The division of the simulations into parallel processes was performed by a threading procedure available by default in the GROMACS program [22].

\section{Calculations of physicochemical properties}

Density $(\rho)$ at different temperatures was obtained by averaging the results from $10 \mathrm{~ns}$ NPT simulations. Pressure in these simulations was set at 1 bar. The results were compared with experimentally obtained densities at different temperatures 
$[23,24]$. The first two points $(293 \mathrm{~K}$ and $298 \mathrm{~K}$ ) required special treatment in order to improve sampling of the configuration space: first a $10 \mathrm{~ns}$ NPT simulation at elevated temperature $(473 \mathrm{~K})$ was performed. Next, ten evenly spaced snapshots were selected from the trajectory. These snapshots were used to initialize simulations at low temperature, and the density was averaged over the ten simulations.

Surface tension $(\gamma)$ was calculated from an NVT ensemble, $100 \mathrm{~ns}$ simulation of a system with the box $z$ dimension extended to approximately three times the original length (box size: $4.026 \mathrm{~nm} \times 4.026 \mathrm{~nm} \times 12.189 \mathrm{~nm}$ ). In this case, the periodic images of simulated liquid were separated by about $8 \mathrm{~nm}$ vacuum in this dimension. After equilibration, the components of the pressure tensor were collected. The surface tension was estimated using the formula [25]

$\gamma=0.5 \cdot \mathrm{L}_{\mathrm{z}}\left(\left\langle\mathrm{P}_{\mathrm{zz}}\right\rangle-\frac{\left\langle\mathrm{P}_{\mathrm{xx}}\right\rangle-\left\langle\mathrm{P}_{\mathrm{yy}}\right\rangle}{2}\right)$

The factor 0.5 is due to the two interfaces present in the system. The results were compared with experimentally obtained surface tension values at different temperatures [23].

Enthalpy of vaporization $\left(\Delta H_{\text {vap }}\right)$ was calculated based on an approach that assumes that the sum of vibrational and kinetic energies is equal for the gas and liquid phase [26]. The formula for enthalpy of vaporization is therefore written as:

$\Delta \mathrm{H}_{\text {vap }}=\mathrm{E}(\mathrm{g})-\mathrm{E}(\mathrm{l})+\mathrm{RT}$

where:

$\mathrm{E}(\mathrm{g})=\mathrm{E}_{\mathrm{dih}}(\mathrm{g})+\mathrm{E}_{\text {intra }}(\mathrm{g})$

$\mathrm{E}(\mathrm{l})=\mathrm{E}_{\mathrm{dih}}(\mathrm{l})+\mathrm{E}_{\text {intra }}(\mathrm{l})+\mathrm{E}_{\text {inter }}(\mathrm{l})$

$E_{\text {dih }}$ describes the dihedral energy term, $E_{\text {intra }}$ is the nonbonding intramolecular interaction energy, and $E_{\text {inter }}$ is the intermolecular interaction energy. Terms ( $g$ ) and (l) refer to the gas and liquid phase, respectively. The gas phase was simulated in a $100 \mathrm{~ns}$ NVT simulation of a single menthol molecule, while the liquid phase was represented as a $10 \mathrm{~ns}$ NVT simulation of a 219-molecule system. The results were compared with experimentally obtained enthalpies of vaporization at different temperatures [27].

Shear viscosity $(\eta)$ was calculated using non-equilibrium periodic perturbation method [28]. A $10 \mathrm{~ns}$ NVT simulation has been performed in a rectangular box with $z$ dimension extended (box size: $3.996 \mathrm{~nm} \times 3.996 \mathrm{~nm} \times 16.382 \mathrm{~nm}$ ). A periodic acceleration $\mathrm{a}_{\mathrm{x}}(\mathrm{z})$ was applied along the $x$-axis

$a_{x}(z)=A \cos \left(\frac{2 \pi}{L_{z}} z\right)$

where $L_{z}$ is the length of the box in the $z$ dimension and $A$ is an arbitrary amplitude parameter. The $A$ parameter has been chosen carefully, to provide the best possible statistics while not moving the system too far from the equilibrium [28]. The simulations were performed for a range of $A$ values $(0.004$, $0.005,0.006,0.01,0.015,0.02,0.03,0.04,0.05,0.06$, $0.07 \mathrm{~nm} / \mathrm{ps}^{2}$ ). To achieve optimal results, the value of $A=$ $0.005 \mathrm{~nm} / \mathrm{ps}^{2}$ was chosen for calculating final viscosity, which was computed according to the formula:

$\eta=\frac{A_{\rho}}{V}\left(\frac{L_{z}}{2 \pi}\right)^{2}$

where $V$ is defined as:

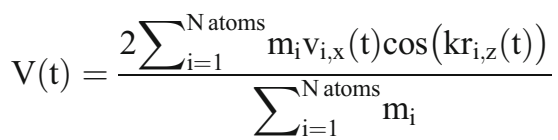

The results were compared with experimentally obtained shear viscosity of liquid menthol at different temperatures [29].

\section{Results and discussion}

It is worth emphasizing that the parameterization strategy and procedure described in "Methods" and discussed here is considered to be standard for acquiring OPLS-AA force field parameters [16]. The rationale behind it is theoretically justified and, importantly, has been proved recently to provide good results for developing imidazolium-based IL force fields [30]. The key feature is its ability to accurately reproduce the physicochemical properties of the studied compound. These properties were therefore used as a basic mean to evaluate the constructed force field.

The bonded parameters - bond and angle interactions, as well as most of the dihedral angle interactions-were therefore taken from the original OPLS-AA force field as they do not contribute much change to those properties. For the dihedral angle interactions, default parameters were also used, with the exception of the angles describing rotation of the hydroxyl and isopropyl group of menthol, which are the only truly labile and asymmetric groups in the molecule. After optimization of the remaining parameters, these angles were tuned to fit the energy profile from restricted Hartree-Fock (RHF) calculations, using the procedure described in "Methods". The energy profiles of the reoptimized angles, as well as those described by original OPLS-AA parameter, are shown in Figs. 1 and 2. These figures show that the tuned dihedral parameters perform much better in describing accurate energetic profiles, making a significant qualitative difference. The whole set of bonded parameters used is shown in Table 1.

Special attention has been given to the parameters describing intermolecular interactions, that is the van der Waals 


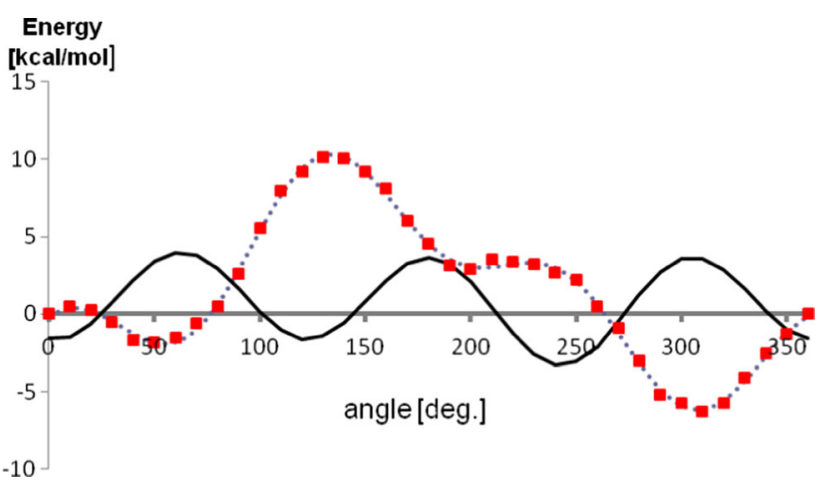

Fig. 1 Profile of the dihedral angle describing the rotation of the hydroxyl group plotted using optimized parameters. Quantum chemical energy is shown using squares, optimized force field energy is shown using dashed line, force field energy calculated with original (nonoptimized) parameters is shown using solid line

parameters and partial atomic charges. The scaling factors for 1-4 Coulomb and van der Waals interactions were conserved from the original force field (amounting to 0.5), the same applies to the exclusion of 1-2 and 1-3 nonbonded interactions. To assess whether or not the van der Waals parameters from the OPLS-AA force field are able to estimate the forces between the molecules correctly, both $\varepsilon$ and $\sigma$ parameters describing the depth of the energy profile and the zeroenergy interatomic distance, respectively, were tuned. Both smaller and larger values of these parameters were tested, the change being applied by scaling the $\varepsilon$ and/or $\sigma$ values for all atoms in the menthol molecule. The original values of van der Waals parameters, proposed in the OPLS-AA force field $[15,16]$, while sufficient for describing the static properties of liquid menthol, had to be adjusted for the calculation of transport properties, namely the shear viscosity of menthol. The depolarization of atomic charges (described below), which was necessary to properly describe the shear viscosity, caused the rest of the calculated properties to deviate from the experimental values. It was found that increasing the $\varepsilon$ value on all atoms up to $118.75 \%$ of the original values was optimal to account for the undesirable changes. The final values of

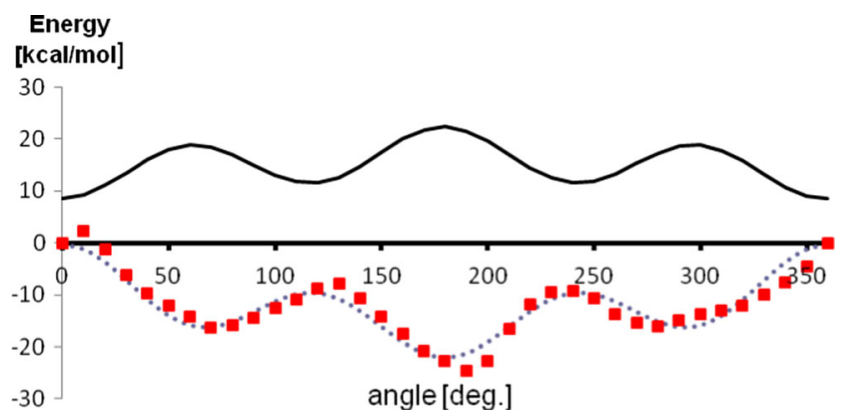

Fig. 2 Profile of the dihedral angle describing rotation of isopropyl group plotted using optimized parameters. Quantum chemical energy is shown using squares, optimized force field energy is shown using dashed line, force field energy calculated with original (non-optimized) parameters is shown using solid line. nonbonding parameters used are presented in Table 1. Simultaneously to the optimization of van der Waals parameters, atomic charges were optimized and re-optimized. To obtain the initial set of partial atomic charges, the geometry of menthol molecule was optimized at the HF/6-31G* level of theory. After that, a single point calculation was performed at the MP2 level, using the aug-cc-pVTZ basis set, with the ftype function excluded, which is a standard procedure for the OPLS-AA force field [16]. The initial charges were calculated according to the CHELPG algorithm [31]. The charges were then rounded up, symmetrized and assigned to atoms. As those charges did not reproduce the properties of menthol (particularly the surface tension of pure menthol) to a satisfactory degree, other sets of charges were prepared and tested using several different methods. These other sets of charges that were tested originated from various computational methods, i.e., NPA [32], AIM [33], and Löwdin [34, 35] methods, as well as the charges proposed by the authors of OPLS-AA force field $[15,16]$. Sets obtained via the NPA method, as well as those proposed by OPLS-AA force field, were additionally tested with increased or decreased van der Waals parameters. The final set of charges comprised the charges proposed by the authors of the OPLS-AA force field, which were obtained using method analogous to described above, but applied to 36 organic liquids $[15,16]$. Those charges were assigned to all atoms with the exception of the hydroxyl group of menthol. For this group, charges obtained from the NPA method [32] were used, resulting in charges more polarized than those from the original force field $(-0.72$ charge on the oxygen atom and 0.45 on the hydrogen atom, before the depolarization described below). These charges were adjusted slightly to keep the molecule neutral. Calculations of the charges using the NPA method were conducted by a method analogous to the one described above - optimization of menthol geometry at the $\mathrm{HF} / 6-31 \mathrm{G}^{*}$ level, followed by a single point calculation at the MP2 level, using the aug-cc-pVTZ(-f) basis set. None of the combinations of charges described above gave better agreement with experimental values than the set chosen for the final force field. It was also found that, to describe the shear viscosity of liquid menthol correctly, charges from the OPLS-AA field needed to be depolarized, by scaling them down to $85 \%$ of their original value. Such a procedure is commonly used when performing IL MD simulations, to account for polarization and charge transfer effects. The reduction of the formal charge (typically by $10 \%, 20 \%$ or even $30 \%$ ) significantly improves the transport properties of IL [14, 36-39]. This issue has been thoroughly studied elsewhere, including ab initio molecular dynamics simulations and quantum chemical calulations [37, 40, 41]. It was found that employing the described scheme for liquid menthol also substantially improves the dynamic properties of the simulated system. The final atomic charges of menthol molecule are presented in Table 2. 
Table 1 Force field parameters for menthol molecule

\begin{tabular}{|c|c|c|c|c|}
\hline Force field parameter & \multicolumn{4}{|l|}{ Value } \\
\hline \multicolumn{5}{|c|}{ Lennard-Jones parameters $[15,16]$} \\
\hline Atom type & \multicolumn{2}{|l|}{$\sigma[\AA]$} & \multicolumn{2}{|c|}{$\varepsilon\left[\mathrm{kJ} \mathrm{mol}^{-1}\right]$} \\
\hline $\mathrm{CT}^{\mathrm{a}}$ & \multicolumn{2}{|l|}{3.50} & \multicolumn{2}{|c|}{0.327921} \\
\hline $\mathrm{HC}^{\mathrm{a}}$ & \multicolumn{2}{|l|}{2.50} & \multicolumn{2}{|l|}{0.149055} \\
\hline $\mathrm{O}$ & \multicolumn{2}{|l|}{3.12} & \multicolumn{2}{|l|}{0.844645} \\
\hline $\mathrm{HO}^{\mathrm{a}}$ & \multicolumn{2}{|l|}{0.00} & \multicolumn{2}{|l|}{0.000000} \\
\hline \multicolumn{5}{|c|}{ Bond stretching parameters $[15,16]$} \\
\hline Bond type & \multicolumn{2}{|l|}{$r_{\mathrm{eq}}[\AA]$} & \multicolumn{2}{|c|}{$K_{\mathrm{r}}\left[10^{-3} \mathrm{~kJ} \mathrm{~mol}^{-1} \mathrm{~nm}^{-2}\right]$} \\
\hline $\mathrm{CT}-\mathrm{CT}$ & \multicolumn{2}{|l|}{1.529} & \multicolumn{2}{|c|}{224.2624} \\
\hline CT-HC & \multicolumn{2}{|l|}{1.090} & \multicolumn{2}{|l|}{284.5120} \\
\hline $\mathrm{CT}-\mathrm{O}$ & \multicolumn{2}{|l|}{1.410} & \multicolumn{2}{|l|}{267.7760} \\
\hline $\mathrm{O}-\mathrm{HO}$ & \multicolumn{2}{|l|}{0.945} & \multicolumn{2}{|l|}{462.7504} \\
\hline \multicolumn{5}{|c|}{ Angle bending parameters $[15,16]$} \\
\hline Angle type & \multicolumn{2}{|l|}{$\theta_{\text {eq }}\left[{ }^{\circ}\right]$} & \multicolumn{2}{|c|}{$K_{\theta}\left[\mathrm{kJ} \mathrm{mol}^{-1} \mathrm{rad}^{-2}\right]$} \\
\hline $\mathrm{HC}-\mathrm{CT}-\mathrm{HC}$ & \multicolumn{2}{|l|}{107.8} & \multicolumn{2}{|c|}{276.144} \\
\hline $\mathrm{HC}-\mathrm{CT}-\mathrm{CT}$ & \multicolumn{2}{|l|}{110.7} & \multicolumn{2}{|l|}{313.800} \\
\hline $\mathrm{CT}-\mathrm{CT}-\mathrm{CT}$ & 112.7 & & 488.273 & \\
\hline $\mathrm{CT}-\mathrm{CT}-\mathrm{O}$ & 109.5 & & 418.400 & \\
\hline $\mathrm{CT}-\mathrm{O}-\mathrm{HO}$ & 108.5 & & 460.240 & \\
\hline $\mathrm{HC}-\mathrm{CT}-\mathrm{O}$ & 109.5 & & 292.880 & \\
\hline Torsional parameters $[\mathrm{kJ} / \mathrm{mc}$ & & & & \\
\hline Dihedral type & $\mathrm{V}_{1}$ & $\mathrm{~V}_{2}$ & $\mathrm{~V}_{3}$ & $\mathrm{~V}_{4}$ \\
\hline CT-CT-CT-CT [16] & -3.3472 & -0.20920 & 0.8368 & 0.0 \\
\hline CT-CT-CT-HC [16] & -7.531 & 0.000 & -1.255 & 0.0 \\
\hline $\mathrm{HC}-\mathrm{CT}-\mathrm{CT}-\mathrm{HC}[16]$ & -7.531 & 0.000 & -1.255 & 0.0 \\
\hline $\mathrm{CT}-\mathrm{CT}-\mathrm{CT}-\mathrm{O}^{\mathrm{b}}$ & 7.159 & -2.092 & 2.774 & 0.0 \\
\hline $\mathrm{HC}-\mathrm{CT}-\mathrm{CT}-\mathrm{O}^{\mathrm{b}}$ & -0.00002 & 0.000 & 1.958 & 0.0 \\
\hline $\mathrm{HC}-\mathrm{CT}-\mathrm{O}-\mathrm{HO}^{\mathrm{b}}$ & 0.000 & 0.000 & 0.000 & 0.0 \\
\hline $\mathrm{CT}-\mathrm{CT}-\mathrm{O}-\mathrm{HO}^{\mathrm{b}}$ & 7.448 & -1.430 & 7.261 & 0.0 \\
\hline Torsional parameters for iso & roup & & & \\
\hline $\mathrm{CT}-\mathrm{CT}-\mathrm{CT}-\mathrm{CT}^{\mathrm{b}}$ & 12.681 & -1.557 & 2.934 & 0.0 \\
\hline $\mathrm{CT}-\mathrm{CT}-\mathrm{CT}-\mathrm{HC} \mathrm{b}^{\mathrm{b}}$ & 0.000 & 0.000 & 0.000 & 0.0 \\
\hline $\mathrm{HC}-\mathrm{CT}-\mathrm{CT}-\mathrm{HC} \mathrm{b}^{\mathrm{b}}$ & 0.000 & 0.000 & 0.000 & 0.0 \\
\hline
\end{tabular}

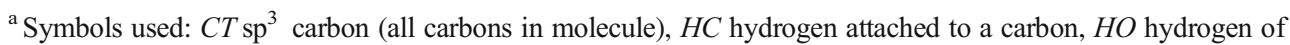
hydroxyl group, $O$ oxygen of hydroxyl group

${ }^{\mathrm{b}}$ Obtained in this work

The summary of all calculated properties is presented in Table 3 and compared with their experimentally measured counterparts. Table 4 provides a comparison of chosen properties calculated with standard OPLS-AA parameters [15, 16] with the same properties calculated using the optimized parameters presented in this work.

The obtained potential allowed the density of pure menthol to be calculated with good accuracy, although the calculated density displays a stronger temperature dependence than the results found experimentally. As described below, this is different from other calculated properties, which tend to show a weaker temperature dependency than the experimental one. It should be mentioned that a better correlation with experimental values can be obtained by using non-depolarized partial atomic charges (that is, charges without scaling down to the $85 \%$ of their original values), at the cost of shear viscosity, which would be several orders of magnitude too high. Therefore, the final set of parameters has to be considered optimal when taking into account static and dynamic properties. For the density of menthol, the best accordance with experiment occurs within the temperature range from $320 \mathrm{~K}$ to $360 \mathrm{~K}$. It should be noted that the available sets of experimental densities for menthol were obtained from two separate sources [23, 24]. It is therefore hard to estimate changes in the 
Table 2 Atomic charges set used in the force field for a menthol molecule

\begin{tabular}{|c|c|c|}
\hline & at. no. & charge \\
\hline \multirow[b]{2}{*}{$(\mathrm{H}=19,20,21)$} & $1,2,4$ & -0.10 \\
\hline & $3,6,9$ & -0.05 \\
\hline \multirow{4}{*}{$(\mathrm{H}=12,13)$} & $7,10,11$ & -0.15 \\
\hline & 5 & 0.18 \\
\hline & 8 & -0.61 \\
\hline & 23 & 0.38 \\
\hline $\begin{array}{c}(\mathrm{H}=14,15) \\
(\mathrm{H}=26,27,28)(\mathrm{H}=29,30,31) \\
(18,22,24=\mathrm{H})\end{array}$ & $\begin{array}{c}12-22,24-31 \\
\text { (Rest of } \\
\text { hydrogens) }\end{array}$ & 0.05 \\
\hline
\end{tabular}

experimental density between $293 \mathrm{~K}$ and $310 \mathrm{~K}$ (see Fig. 3). Calculations of the surface tension of pure menthol turned out to be computationally demanding. In order to avoid the uncertainties of the surface tension values being too high, the simulation time had to be extended to $100 \mathrm{~ns}$ (compared to $10 \mathrm{~ns}$ simulations for calculation of other properties). This is associated with the character of the calculated property; since the surface tension is obtained based on the components of pressure tensor, it is affected strongly by large oscillations in pressure, which are to be expected. The obtained force field is capable of producing surface tension values with good accordance to experimental values [23]; the values obtained are from $2 \times 10^{-3}$ to $3 \times 10^{-3} \mathrm{~N} \mathrm{~m}^{-1}$ lower than the experimental ones (Fig. 3). The calculated values of the enthalpy of vaporization also remain in good accordance with experimental results [27]. While the calculated enthalpies of vaporization

Table 3 Comparison of experimental and calculated properties of liquid menthol for the obtained force field.

\begin{tabular}{|c|c|c|c|c|c|}
\hline & & Experimental & Calculated & $\mathrm{SD}^{\mathrm{a}}$ & Deviation from experimental value ${ }^{b}$ \\
\hline & & \multicolumn{3}{|c|}{ Density $\left[\mathrm{kg} \mathrm{m}^{-3}\right]$} & {$[\%]$} \\
\hline \multirow[t]{6}{*}{$\mathrm{T}[\mathrm{K}]$} & 293 & $890[24]$ & 921.1 & 1.1 & 3.49 \\
\hline & 298 & $923.6[24]$ & 917.3 & 1.2 & 0.68 \\
\hline & 318 & 889 [23] & 902.0 & 3.4 & 1.46 \\
\hline & 353 & 877 [23] & 872.1 & 3.5 & 0.56 \\
\hline & 393 & $865.1[23]$ & 836.4 & 3.8 & 3.32 \\
\hline & & \multicolumn{3}{|c|}{ Surface tension $\left[\mathrm{mN} \mathrm{m}^{-1}\right]$} & {$[\%]$} \\
\hline \multirow[t]{6}{*}{$\mathrm{T}[\mathrm{K}]$} & 313 & $31.61[23]$ & 28.6 & 8.2 & 9.52 \\
\hline & 333 & $29.2[23]$ & 27.1 & 6.5 & 7.19 \\
\hline & 353 & $27.82[23]$ & 24.9 & 5.4 & 10.50 \\
\hline & 373 & $25.62[23]$ & 22.7 & 3.6 & 11.40 \\
\hline & 393 & $23.75[23]$ & 20.6 & 3.0 & 13.26 \\
\hline & & \multicolumn{3}{|c|}{ Enthalpy of vaporization $\left[\mathrm{kJ} \mathrm{mol}^{-1}\right]$} & {$[\%]$} \\
\hline \multirow[t]{4}{*}{$\mathrm{T}[\mathrm{K}]$} & 382 & $56.92[27]$ & 52.8 & 5.7 & 7.24 \\
\hline & 421 & $53.42[27]$ & 51.1 & 4.8 & 4.34 \\
\hline & 473 & $48.42[27]$ & 49.5 & 5.5 & 2.23 \\
\hline & & \multicolumn{3}{|c|}{ Shear viscosity $\left[\mathrm{mPa} \mathrm{s}^{-1}\right]$} & {$[\%]$} \\
\hline \multirow[t]{3}{*}{$\mathrm{T}[\mathrm{K}]$} & 320 & $10.8[29]$ & 5.9 & 1.2 & 45.37 \\
\hline & 350 & $2.3[29]$ & 3.8 & 0.58 & 65.22 \\
\hline & 380 & 0.83 [29] & 2.8 & 0.36 & 237.4 \\
\hline
\end{tabular}

${ }^{\text {a }}$ Standard deviation of the calculated values

${ }^{\mathrm{b}}$ Percentage deviation of the results calculated with obtained force field from the corresponding experimental values 
Table 4 Density and enthalpy of vaporization of liquid menthol calculated using standard and optimized parameters, in comparison with experimental values

\begin{tabular}{|c|c|c|c|c|c|c|}
\hline & & Experimental & Standard parameters & Relative error [\%] & Optimized parameters & Relative error [\%] \\
\hline \multicolumn{7}{|c|}{ Density $\left[\mathrm{kg} \mathrm{m}^{-3}\right]$} \\
\hline \multirow[t]{3}{*}{$\mathrm{T}[\mathrm{K}]$} & 293 & $890[24]$ & 896.1 & 0.7 & 921.1 & 3.5 \\
\hline & 318 & 889 [23] & 875.0 & 1.6 & 902.0 & 3.4 \\
\hline & 393 & 865.1 [23] & 801.8 & 7.3 & 836.4 & 3.8 \\
\hline \multicolumn{7}{|c|}{ Enthalpy of vaporization $\left[\mathrm{kJ} \mathrm{mol}^{-1}\right]$} \\
\hline \multirow[t]{3}{*}{$\mathrm{T}[\mathrm{K}]$} & 382 & $56.92[27]$ & 31.9 & 44.0 & 52.8 & 7.2 \\
\hline & 421 & $53.42[27]$ & 29.7 & 44.4 & 51.1 & 4.3 \\
\hline & 473 & $48.42[27]$ & 26.7 & 44.9 & 49.5 & 2.2 \\
\hline
\end{tabular}
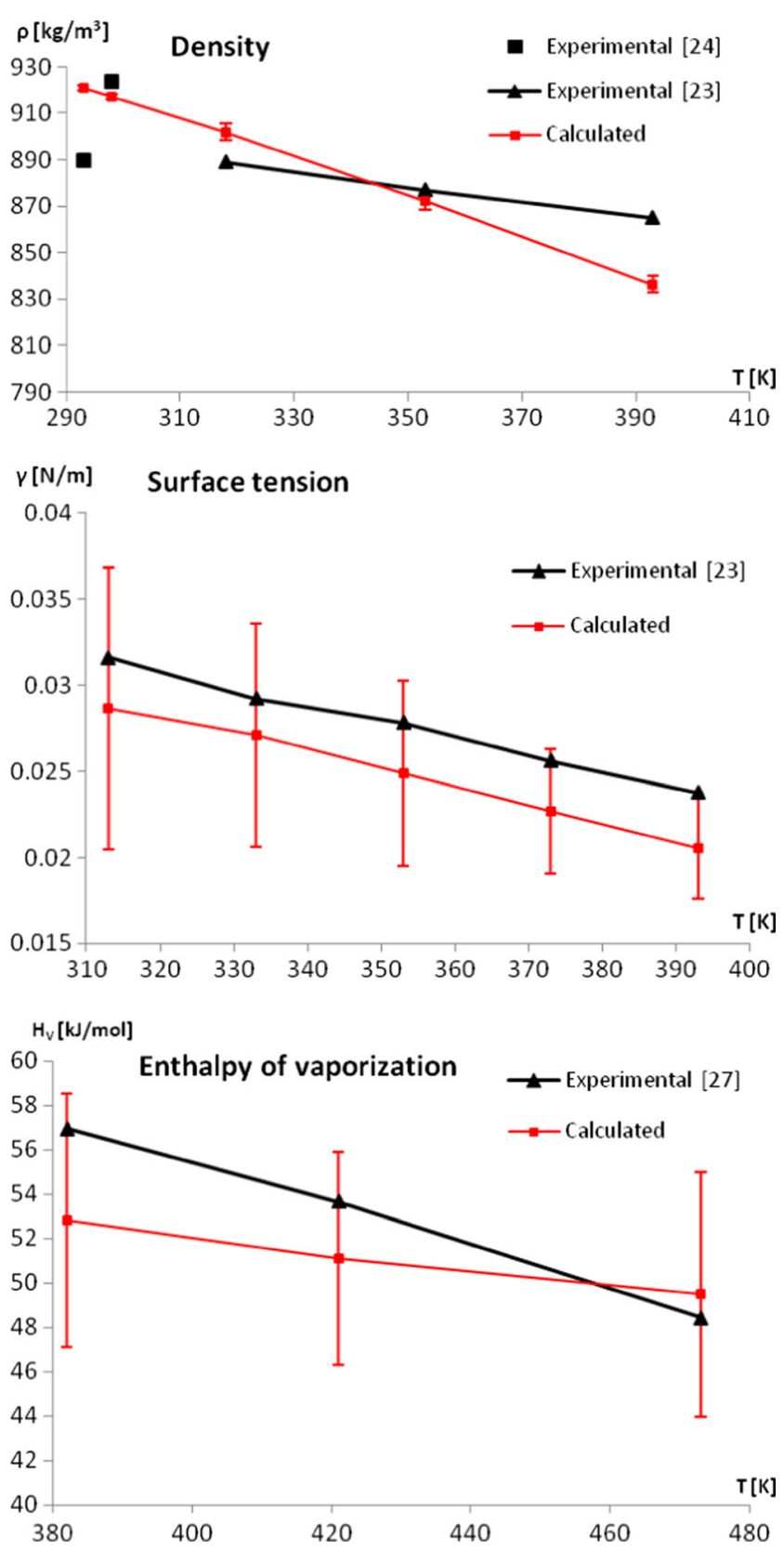

Fig. 3 Comparison between calculated and experimental [23, 24, 27] thermodynamic properties of pure menthol stay within the proper range of values, the temperature dependence of this property is weaker than indicated by experiment, which leads to slightly underestimated values at lower temperatures. This property is prone to large uncertainty values, due to the large variation associated with gas phase simulations; the gas phase state is modelled by the simulation of a single menthol molecule. It is therefore recommended to perform longer simulations for this phase (in this work $100 \mathrm{~ns}$ simulations were performed). The shear viscosity of a liquid, being a dynamic property, is one of the most important characteristics that should validate the force field, especially for compounds that demand more carefully adjusted parameters [14]. The arbitrarily chosen parameter A (the amplitude of the induced fluctuations) turns out to be of great importance for calculating the viscosity of menthol. Only a narrow range of tested amplitudes allowed to calculate the investigated property reliably. Values of A higher than $0.06 \mathrm{~nm} / \mathrm{ps}^{2}$ caused the viscosity to decrease rapidly, indicating that the system was too strongly perturbed, and diverged from the metastable state of non-equilibrium dynamics. Simultaneously, the lower amplitudes are not capable of inducing an organized motion in the system (compared to normal thermal motions), the friction is too low, and the uncertainties become too high; decreasing A from $0.06 \mathrm{~nm} \mathrm{ps}^{-2}$ to $0.04 \mathrm{~nm} \mathrm{ps}^{-2}$ can increase the

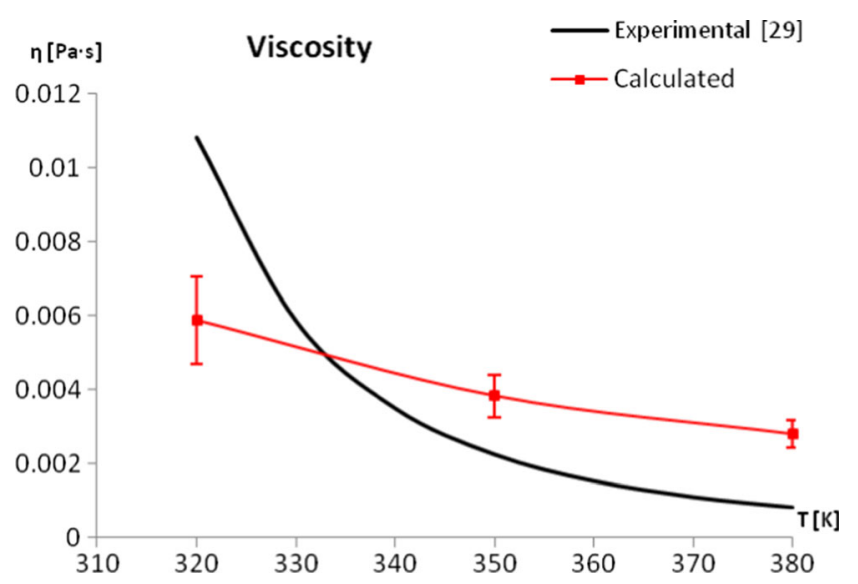

Fig. 4 Comparison between calculated and experimental [29] shear viscosity of pure menthol. The experimental value is represented as a calculated function within the recommended temperature range [29] 
calculated standard deviation by as much as $59 \%$ in lower temperatures. Therefore, the final value of $A=0.05 \mathrm{~nm} \mathrm{ps}^{-2}$ was chosen as a compromise, which resulted in relatively low values of calculated standard deviations and did not disturb the system too much. The obtained force field allows the shear viscosity of liquid menthol to be reproduced well within the range of the experimental values [29] (Fig. 4, Table 3), although it is not able to correctly describe the changes of viscosity in the whole temperature range. The problem could possibly be remedied by including polarization terms in the force field. A very similar unsatisfactory temperature dependence of the calculated viscosity was observed for the MD of liquid water [42], with the addition of polarization terms as the suggested solution. It has also been confirmed that including polarization effects is important for the proper description of the temperature-dependent properties of organic liquids such as ethylene glycol [43]. It was, however, beyond the aim of this study to investigate this solution, as one of our main goals was to keep the force field compatible with the standard of the OPLS-AA force field.

\section{Conclusions}

In this work, a force field for the MD simulations of liquid menthol was constructed, evaluated and optimized. The presented force field is fully compatible with the OPLS-AA force field and can possibly be used together with other potentials adhering to this standard. Furthermore, the proposed potential avoids introducing any non-standard energy terms or scaling factors inconsistent with the OPLS-AA standard. The potential transferability of the developed force field was additionally tested by performing several MD simulations of mentholsubstituted, imidazolium-based ILs (unpublished data), resulting in a reliable description of their densities. The potential describing rotation of hydroxyl and isopropyl groups was fitted to the quantum chemical profiles, which significantly improved the torsional potentials. The force field was validated by calculating various physicochemical properties of liquid menthol. Both static (density, surface tension, enthalpy of vaporization) and transport (shear viscosity) properties were calculated. This range of investigated properties should be considered an advantage of the developed force field, as it was aimed at providing versatility and good performance while assessing many different characteristic simultaneously. While several combinations of parameters were tested, the final version of the force field was judged to be optimal, that is, to describe all the aforementioned properties with the best accordance with their respective experimental counterparts. The force field performs well for the chosen properties, although it suffers a bit when temperature dependence is considered. However, the force field should be applied with caution when reproducing transport properties or those based on the gas phase of the investigated compound considering the issues discussed above. It is worth emphasizing that the force field describes the viscosity of menthol with acceptable accuracy; this property is considered to be difficult to reproduce in MD simulations and, as such, is a good measure of the quality of tested parameters.

Acknowledgments This research was financed by the National Science Center (Poland) grant no. 2011/01/B/ST/06659. Calculations were performed at the Wrocław Center for Networking and Supercomputing (grant No. 012).

Open Access This article is distributed under the terms of the Creative Commons Attribution 4.0 International License (http:// creativecommons.org/licenses/by/4.0/), which permits unrestricted use, distribution, and reproduction in any medium, provided you give appropriate credit to the original author(s) and the source, provide a link to the Creative Commons license, and indicate if changes were made.

\section{References}

1. Patel T, Ishiuji Y, Yosipovitch G (2007) Menthol: a refreshing look at this ancient compound. J Am Acad Dermatol 57(5):873-878

2. Farco JA, Grundmann O (2013) Menthol—pharmacology of an important naturally medicinal "cool". Mini-Rev Med Chem 13: 124-131

3. Oertling H, Reckziegel A, Surburg H, Bertram HJ (2007) Applications of menthol in synthetic chemistry. Chem Rev 107: 2136-2164

4. Pernak J, Feder - Kubis J (2005) Synthesis and properties of chiral ammonium-based ionic liquids. Chem Eur J 11:4441-4449

5. Pernak J, Feder - Kubis J (2006) Chiral Pyridinium-based ionic liquids containing the $(1 \mathrm{R}, 2 \mathrm{~S}, 5 \mathrm{R})-(-)$-Menthyl group. Tetrahedron: Assymetry 17:1728-1737

6. Pernak J, Feder-Kubis J, Cieniecka-Rosłonkiewicz A, Fischmeister C, Griffin ST, Rogers RD (2007) Synthesis and properties of Chiral Imidazolium ionic liquids with a (1R,2S,5R)-(-)-Menthoxymethyl substituent. New J Chem 31:879-892

7. Bicchi C, Brunelli C, Cravotto G (2003) Cyclodextrin derivatives in Enantiomer GC separation of volatiles. Part XXI: complexation of some Terpenoids with 2-O-Acetyl-3-O-Methyl- and 2-O-Methyl-3O-Acetyl-6-O-T-Hexyldimethylsilyl-C-Cyclodextrins: molecular mechanics and molecular dynamics. J Sep Sci 26:1479-1490

8. Ruggera JF, Merla AB, Vetere V, Casella ML (2010) Toward a rational design of enantioselective heterogenous catalysts: modeling of chiral organotin precursors. J Mol Str Theochem 953:91-97

9. Pedretti A, Labozzetta A, Lo Monte M, Beccari AR, Moriconi A, Vistoli G (2011) Exploring the activation mechanism of TRPM8 channel by targeted MD simulations. Biochem Biophys Res Commun 414:14-19

10. Raffaini G, Ganazzoli F, Malpezzi L, Fuganti C, Fronza G, Panzeri W, Mele A (2009) Validating a strategy for molecular dynamics simulations of Cyclodextrin inclusion complexes through singlecrystal X-ray and NMR experimental data: a case study. J Phys Chem B 113:9110-9122

11. Smith WB, Amezcua C (1998) NME versus molecular modelling: Menthone and Isomenthone. Magn Reson Chem 36:S3-S10

12. Härtner J, Reincheid UM (2008) Conformational analysis of Methol Diasteromers by NMR and DTF computation. J Mol Str 872:145-149 
13. McCann JL, Rauk A, Wieser H (1998) A conformational study of (1S, 2R, 5S)-(+)-menthol using vibrational circular dichroism spectroscopy. Canadian J Chem 76(30):274-283

14. Maginn EJ (2009) Molecular simulation of ionic liquids: current status and future opportunities. J Phys Condens Matter 21(37): 373101

15. Jorgensen WL, Tirado-Rives J (1988) The OPLS potential for proteins. Energy minimizations for crystals of cyclic Peptidesand Crambin. J Am Chem Soc 110(6):1657-1666

16. Jorgensen WL, Maxwell DS, Tirado-Rives J (1996) Development and testing of the OPLS all-atom force field on conformational energetics and properties of organic liquids. J Am Chem Soc 118(45):11225-11236

17. Berendsen HJC, Postma JPM, van Gunsteren WF, DiNola A, Haak JR (1984) Molecular-dynamics with coupling to an external bath. J Chem Phys 81(8):3684-3690

18. Nosé S (1984) A molecular-dynamics method for simulations in the canonical ensemble. Mol Phys 52(2):255-268

19. Hoover WG (1985) Canonical dynamics-equilibrium phase-space distribution. Phys Rev A 3:1695-1697

20. Parrinello M, Rahman A (1981) Polymorphic transitions in singlecrystals - a new molecular dynamics method. J Appl Phys 52(12): $7182-7190$

21. Hess B, Bekker H, Berendsen HJC, Fraaije JGEM (1997) LINCS: a linear constraint solver for molecular simulations. J Comput Chem 18(12): 1463-1472

22. Hess B, Kutzner C, van der Spoel D, Lindahl E (2008) GROMACS 4: algorithms for highly efficient, load-balanced, and scalable molecular simulation. J Chem Theory Comput 4(3):435-447

23. Popov G, Kolarov K, Velchev K, Manolov KR (1977) Physical characteristics of the main components of Bulgarian essential oils. Monatshefte fuer Chemie 108(1):159-161

24. Kozlov NG, Pehk T, Valimae T (1981) Reduction Amination of LMenthol by aliphatic Nitriles. Khmiya Prirodnykh Soedinenii, 3: 312-317. [data from REAXYS® database https://www.reaxys. $\mathrm{com} /]$

25. Szefczyk B, Cordeiro M, Natalia DS (2011) Physical properties at the base for the development of an all-atom force field for ethylene glycol. J Phys Chem B 115(2):3013-3019

26. Jorgensen WL, Madura JD, Swenson CJ (1984) Optimized intermolecular potential functions for liquid hydrocarbons. J Am Chem Soc 22:6638-6646

27. Cramer JSN (1943) Vapor-pressure measurements on some organic substances. Recueil des Travaux Chimiques des Pays-Bas et de la Belgique, 62: 606-10 [data from REAXYS® database https://www. reaxys.com/].

28. Hess B (2002) Determining the shear viscosity of model liquids from molecular dynamics simulations. J Chem Phys 116(1):209217
29. Viswanath DS, Ghosh TK, Prasad DHL, Dutt NVK, Rani KY (2007) CCorrelations and Estimation of Pure Liquids Viscosity. In: Viswanath DS, Ghosh TK, Prasad DHL, Dutt NVK, Rani KY (eds) Viscosity of Liquids. Theory, Estimation, Experiment, and Data. Springer, Dordrecht, pp 135-405

30. Sambasivarao SV, Acevedo O (2009) Development of OPLS-AA force field parameters for 68 unique ionic liquids. J Chem Theory Comput 5:1038-1050

31. Breneman CM, Wiberg KB (1990) Determining atom-centered monopoles from molecular electrostatic potentials. The need for high sampling density in formamide conformational analysis. J Comput Chem 11:361-373

32. Reed AE, Weinstock RB, Weinhold F (1983) Natural population analysis. J Chem Phys 83(2):735-746

33. Bader RF (1990) Atoms in molecules: a quantum theory. Oxford University Press, New York

34. Löwdin PO (1970) On the nonorthogonality problem. Adv Quantum Chem 5:185-189

35. Cusachs LC, Politzer P (1968) On the problem of defining the charge on an atom in a molecule. Chem Phys Lett 1:529

36. Bhargava BL, Balasurbamanian S (2007) Refined potential for atomistic simulations of ionic liquid [bmim][PF6]. J Chem Phys 127: 114510

37. Morrow TI, Maginn EJ (2002) Molecular dynamics study of the ionic liquid 1-n-Butyl-3-methylimidazolium Hexafluorophosphate. J Phys Chem B 106:12807-12813

38. Youngs TGA, Hardacre C (2008) Application of static charge transfer within and ionic-liquid force field and its effect on structure and dynamics. ChemPhysChem 9(11):1548-1558

39. Sieffert N, Wipff J (2006) The [BMI][Tf2N] ionic liquid/water binary system: a molceular dynamics study of phase separation and of the liquid-liquid interface. Phys Chem B 110(26):13076-13085.42

40. Schmidt J, Krekeler C, Dommert F, Zhao YY, Berger R, Delle Site L, Holm C (2010) Ionic charge reduction and atomic partial charges from first-principles calculations of 1,3-Dimethylimidazolim chloride. J Phys Chem B 114(18):6150-6155.43

41. Wendler K, Zahn S, Dommert F, Berger R, Holm C, Kirchner B, Delle SL (2011) Locality and fluctuations: trends in ImidazoliumBased ionic liquids and beyond. J Chem Theor Comput 7(10): 3040-3044

42. Medina JS, Prosmiti R, Villarreal P, Delgado-Barrio G, Winter G, González B, Alemán JV, Collado C (2011) Molecular dynamics simulations of rigid and flexible water models: temperature dependence of viscosity. Chem Phys 388:9-18

43. Geerke DP, van Gunsteren WF (2007) The performance of nonpolarizable and polarizable force-filed parameter sets for ethylane glycol in molecular dynamics simulations of the pure liquid and its aqueous mixtures. Mol Phys 105(13-14):1861-1881 